\title{
Fast Jacobi-type Algorithm for Computing Distances Between Linear Dynamical Systems
}

\author{
Nicolas D. Jimenez Bijan Afsari René Vidal \\ Center for Imaging Science, Johns Hopkins University \\ $\{$ njimenez, bijan, rvidal\}ecis.jhu.edu
}

\begin{abstract}
A novel metric between linear dynamical systems, the alignment distance, was recently introduced, with promising results in many computer vision tasks. The computation of the alignment distance requires a minimization over the orthogonal group. In this paper, we present a fast and accurate Jacobi-type algorithm that solves this problem. Each step of the algorithm is equivalent to finding the roots of a quartic polynomial. We show that this rooting may be done efficiently and accurately using a careful implementation of Ferrari's classical closedform solution for quartic polynomials. For linear systems with orders that commonly arise in computer vision scenarios, our algorithm is roughly twenty times faster than a fast Riemannian gradient descent algorithm implementation and has comparable accuracy.
\end{abstract}

\section{INTRODUCTION}

A distance between two linear dynamical systems (LDSs) is a useful notion and several attempts have been made to define distances between LDSs. In control theory such attempts go back to 1970's (e.g., [14]) for applications in system identification. More recently, interest in distances between LDSs has resurfaced for applications in pattern recognition (e.g., classification and clustering) for high dimensional time-series data. A particular example is the automatic classification of videos of human activities such as walking, running, and jumping or more even more complicated human activities [7] or natural dynamic scenes [9]. A common approach to this problem is to, first, estimate LDS parameters to model observed video sequences (or features extracted thereof) and then to compare the learnt LDSs via an appropriate distance (see e.g., [4], [7] and references therein). Notice that such a distance is a versatile tool and can be used to perform more general statistical analysis (e.g., to compute averages).

In the above mentioned setting one usually is led to compare two LDSs of the same fixed size (i.e., numbers of inputs and outputs) and the same fixed order, and hence one would like to define a distance between such LDSs. In [4] an example of such a distance called the alignment distance was introduced for a specific class of LDSs (see [4] and $\S$ II for details) ${ }^{1}$ The main idea behind the alignment distance is that an LDS has an equivalent class of state-space realizations and two such realizations cannot be compared

\footnotetext{
${ }^{1}$ In this paper, we introduce our algorithm with respect to this specific class of LDSs. However, the alignment distance can be extended to more general classes of LDSs including stochastic systems (the details of this will appear in forthcoming papers [5], [6]). Interestingly, the algorithm presented here is still applicable (with little or no modification) to all these general classes.
}

directly. Recall that the equivalent realizations are related to each other via linear change of basis in the state-space domain. Hence, in computing the alignment distance one, first, solves the (realization) alignment problem to align (in a specific sense) the given realizations of two LDSs and then compares the aligned realizations (see $\S$ II for more details).

The alignment problem is, in fact, a minimization problem over a group of orthogonal matrices for which in [4] a Riemannian gradient descent algorithm was introduced. As a better alternative, in this paper, we introduce a fast and accurate Jacobi-type algorithm for computing alignment distances. Our Jacobi-type algorithm solves the multidimensional optimization problem by solving a sequence of 1dimensional subproblems. These 1-dimensional subproblems can be solved by prudent use of closed form solutions for quartic polynomials. In computer vision applications, such as classification and clustering of video sequences, one deals with large numbers of systems. Thus, fast computation of these distances is extremely desirable for ensuring the feasibility of clustering and classification tasks. Our method is significantly faster than the Riemannian gradient descent, as we will show. Besides that, our algorithm is step-size free (a significant merit over the gradient descent algorithm), and moreover, as our experiments show, it is more likely than the gradient descent algorithm to find global minimizers. Although control applications are not the focus of this paper, we mention that, the alignment distance could be potentially used in system identification or robust control applications.

This paper is organized as follows: In \$II we briefly review the methodology needed to define the alignment distances. In $8 \mathrm{III}$ the details of the proposed algorithm are described. In $\mathrm{IV}$ numerical simulations are presented to validate the performance of our algorithms. Efficient MATLAB implementations of these algorithms may be downloaded from http: / /www.vision.jhu.edu/downloads/.

\section{BRIEF INTRODUCTION TO THE ALIGNMENT DiSTANCE}

We now briefly describe the theoretical basis for the alignment distance for deterministic LDSs (more details, extensions, and proofs will appear in [5], [6]). Our treatment is based on intuition, but it can be made rigorous via the machinery of differential geometry, specifically, the theory of fiber bundles. Consider a discrete-time LDS $M$ of order $n$ and size $(p, m)$ (i.e., $m$-dimensional input and $p$-dimensional 
output) described by:

$$
\begin{aligned}
& \mathbf{x}_{t}=A \mathbf{x}_{t-1}+B \mathbf{v}_{t} \\
& \mathbf{y}_{t}=C \mathbf{x}_{t}
\end{aligned}
$$

where $R=(A, B, C) \in \widetilde{\mathcal{L}}_{m, n, p}=\mathbb{R}^{n \times n} \times \mathbb{R}^{n \times m} \times \mathbb{R}^{p \times n}$ is a realization of $M$. Here, $\mathbf{v}_{t}$ is the input which we assume to be deterministic. We call $\widetilde{\mathcal{L}}_{m, n, p}$ a realization space and we explicitly distinguish it from $\mathcal{L}_{m, n, p}$ the space of LDSs of size $(p, m)$ and order $n$. The space $\mathcal{L}_{m, n, p}$ is comprised of equivalent classes of realizations where $(A, B, C)$ is equivalent to

$$
P \circ(A, B, C)=\left(P^{-1} A P, B^{-1} P, C P\right),
$$

for any $P \in G L(n)$, where $G L(n)$ is the group of nonsingular $n \times n$ matrices. Due to this equivalence relation, in order to compare two systems $M_{1}$ and $M_{2}$ one cannot simply compare their arbitrary realizations $R_{1}$ and $R_{2}$. Instead, one needs first to optimally align the two realizations (i.e., bring them closest together by sliding them along their respective equivalence classes). However, due to certain theoretical and computational difficulties, mostly stemming from noncompactness of $G L(n)$, such an alignment is hard to define and perform. Instead, we propose first to standardize the realizations and then align them. By standardization, intuitively, we mean a step in which the non-compact part of $G L(n)$ is thrown out and only its maximal compact subgroup namely the group of $n \times n$ matrices $O(n)$ is kept. It can be shown that standardization is possible for a large class of LDSs; however, here, we only consider it in relation to a specific class of LDSs which we call tall and full rank. This class appears naturally as the output of a system identification algorithm popular in video sequence analysis (see [4], [9]). Let $\widetilde{\mathcal{L}^{\mathrm{tC}}}{ }_{m, n, p}=\left\{R \in \widetilde{\mathcal{L}}_{m, n, p} \mid \operatorname{rank}(C)=n\right\}$, i.e., realizations in which $C$ is of full column rank. Denote the corresponding space of LDSs by $\mathcal{L}_{m, n, p}^{\mathrm{tC}}$. A simple standardized space related to $\mathcal{L}_{m, n, p}^{\mathrm{tC}}$ is $\widetilde{\mathcal{O}}_{m, n, p}^{\mathrm{tC}}=\{R \in$ $\left.\widetilde{\mathcal{L}}_{m, n, p}^{\mathrm{tC}} \mid C^{\top} C=I_{n}\right\}$, where $I_{n}$ is the $n \times n$ identity matrix and ${ }^{\top}$ denotes matrix transpose. Notice that any realization in $\mathcal{L}_{m, n, p}^{\mathrm{tC}}$ can be standardized e.g., via SVD of $C$. Moreover, on $\widetilde{\mathcal{O L}}_{m, n, p}, G L(n)$ acts only through its subgroup $O(n)$, since $P \circ R \in \widetilde{\mathcal{O L}}_{m, n, p}^{\mathrm{tC}}$ implies $P \in O(n)$ for any $P \in G L(n)$ and $R \in \widetilde{\mathcal{O}}_{m, n, p}{ }^{\mathrm{tC}}$.

Upon standardization, aligning the realizations and comparison of LDSs become straightforward. More specifi-

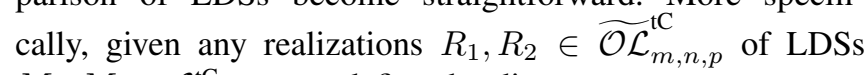
$M_{1}, M_{2} \in \mathcal{L}_{m, n, p}^{\mathrm{tC}}$, we define the distance

$$
d^{2}\left(M_{1}, M_{2}\right)=\min _{Q \in O(n)} d_{F}^{2}\left(Q \circ R_{1}, R_{2}\right),
$$

where $d_{F}$ is the following Frobenius norm based distance on $\widetilde{\mathcal{O}}_{m, n, p}{ }^{\mathrm{tC}}$

$\tilde{d}_{F}^{2}\left(R_{1}, R_{2}\right)=\lambda_{A}\left\|A_{1}-A_{2}\right\|_{F}^{2}+\lambda_{B}\left\|B_{1}-B_{2}\right\|_{F}^{2}+\lambda_{C}\left\|C_{1}-C_{2}\right\|_{F}^{2}$,
The minimization in (3) is called the (realization) alignment problem and can be explicitly expressed as $d^{2}\left(M_{1}, M_{2}\right)=$ $\min _{Q \in O(n)} f_{1}\left(Q ; R_{1}, R_{2}\right)$, where $f: O(n) \rightarrow \mathbb{R}$ is:

$$
\begin{aligned}
f\left(Q ; R_{1}, R_{2}\right)= & \lambda_{A}\left\|Q^{\top} A_{1} Q-A_{2}\right\|_{F}^{2}+\lambda_{B}\left\|Q^{\top} B_{1}-B_{2}\right\|_{F}^{2} \\
& +\lambda_{C}\left\|C_{1} Q-C_{2}\right\|_{F}^{2} .
\end{aligned}
$$

Note that the parameters $\lambda_{A}, \lambda_{B}, \lambda_{C}$ determine the relative contributions of the $A, B, C$ matrices to the cost function. As such, they allow the possibility of tuning the metric for a particular application.

\section{JACOBI-TYPE Algorithm FOR COMPUting THE ALIGNMENT DistANCE}

The Jacobi eigenvalue algorithm is a well established method in numerical linear algebra [12]. More generally Jacobi-type methods have been used for minimizing functions defined on $O(n)$ (e.g., joint diagonalization). Such methods are essentially coordinate descent optimizations on the manifold $O(n)$, i.e., one performs a sequence of 1-dimensional minimizations along fixed directions. These directions are, in fact, geodesics (equivalent of straight line on $O(n)$ ) along the components of an orthogonal basis of the tangent space at each point. The amount of descent is preferably determined by finding the exact solution to the 1-dimensional minimization sub-problem. Due to the specific selection of descent directions, the 1-dimensional sub-problems are usually easy to solve, and in fact a global minimizer can often be found. A major benefit of Jacobi-type methods is their local quadratic rate of convergence [13]. These methods can be extended to groups other than $O(n)$, including non-compact groups (see e.g., [3], [13], [15]).

\section{A. Jacobi-type Algorithm and Lagrangian for Minimizing $f$}

First, recall that $O(n)$ has two connected components: $S O(n)$, which is comprised of orthogonal matrices of determinant +1 , and $O^{-}(n)$, whose elements have determinant -1 . To minimize $f\left(Q ; R_{1}, R_{2}\right)$ (see $(5)$ ), we choose an initial value for $Q$ (once in $S O(n)$ and once in $O(n)$ ) and update $R_{1}$ as follows: $R_{1} \leftarrow Q \circ R_{1}$. Given this initialization, we then perform updates of the form

$$
Q \leftarrow Q Q_{p q}\left(\theta_{p q}\right) \text { and } R_{1} \leftarrow Q_{p q}\left(\theta_{p q}\right) \circ R_{1},
$$

where $Q_{p q}(\theta)$ is the Givens rotation matrix

$$
Q_{p q}(\theta)=\left[\begin{array}{ccccccc}
1 & \cdots & \downarrow & \cdots & \downarrow & \cdots & 0 \\
\vdots & \ddots & \vdots & & \vdots & & \vdots \\
p \rightarrow & \cdots & c & \cdots & -s & \cdots & 0 \\
\vdots & & \vdots & \ddots & \vdots & & \vdots \\
q \rightarrow & \cdots & s & \cdots & c & \cdots & 0 \\
\vdots & & \vdots & & \vdots & \ddots & \vdots \\
0 & \cdots & 0 & \cdots & 0 & \cdots & 1
\end{array}\right]
$$

with $c=\cos (\theta)$ and $s=\sin (\theta)$ with $\theta \in(-\pi, \pi]$. We find $\theta_{p q}$ via $\min _{\theta} f\left(Q_{p q}(\theta) ; R_{1}, R_{2}\right)$. However, it is more convenient to do minimization over $s, c$ subject to the constraint $s^{2}+c^{2}=1$. The algorithm usually begins from 
$(p, q)=(1,2)$ and progresses by sweeping through all index pairs $(p, q)$ such that $q>p$ (this is called one sweep) and then repeats the process till convergence. In view of our discussion above, we mention that $\theta \mapsto Q_{p q}(\theta)=\exp \left(\theta \Omega_{p q}\right)$ is the geodesic starting from $I_{n}$ along the direction of the skew-symmetric tangent vector $\Omega_{p q}$, where exp is the matrix exponential $\left(\Omega_{p q}\right.$ is found by simply setting the diagonal of $Q_{p q}$ to zero and replacing $s$ by 1 .)

Next, we turn to the 1-dimensional minimization problem $\min _{c^{2}+s^{2}=1} f\left(Q_{p q}(c, s) ; R_{1}, R_{2}\right)$. Clearly, the minimization of $f$ (see (5)) is equivalent to maximizing:

$$
f\left(Q_{p q}(c, s)\right)=\lambda_{A} \operatorname{tr}\left(Q^{\top} A_{1}^{\top} Q A_{2}\right)+\operatorname{tr}\left(Q^{\top} D\right)
$$

where $D=\lambda_{B}\left(B_{1} B_{2}^{\top}\right)+\lambda_{C}\left(C_{1}^{\top} C_{2}\right)$ (note that $Q^{\top} Q=I_{n}$ ). We denote the elements of the defined matrices as follows: $\left[A_{1}\right]_{i j}=a_{i j},\left[A_{2}\right]_{i j}=a_{i j}^{\prime},[D]_{i j}=d_{i j}$. A straightforward computation shows that

$$
f\left(Q_{p q}(c, s)\right)=k_{0} c^{2}+k_{1} s^{2}+k_{2} c s+k_{3} c+k_{4} s,
$$

where

$$
\begin{aligned}
k_{0}= & \lambda_{A}\left[a_{p p} a_{p p}^{\prime}+a_{p q} a_{p q}^{\prime}+a_{q q} a_{q q}^{\prime}+a_{q p} a_{q p}^{\prime}\right] \\
k_{1}= & \lambda_{A}\left[a_{p p} a_{q q}^{\prime}+a_{q q} a_{p p}^{\prime}-a_{p q} a_{q p}^{\prime}-a_{q p} a_{p q}^{\prime}\right] \\
k_{2}= & \lambda_{A}\left[a_{p p}\left(-a_{q p}^{\prime}-a_{p q}^{\prime}\right)+a_{p q}\left(a_{p p}^{\prime}-a_{q q}^{\prime}\right)+\right. \\
& \left.a_{q p}\left(a_{p p}^{\prime}-a_{q q}^{\prime}\right)+a_{q q}\left(a_{p q}^{\prime}+a_{q p}^{\prime}\right)\right] \\
k_{3}= & d_{p p}+d_{q q}+\lambda_{A} \sum_{j \neq p, q} a_{j p} a_{j p}^{\prime}+a_{j q} a_{j q}^{\prime}+a_{p j} a_{p j}^{\prime}+a_{q j} a_{q j}^{\prime} \\
k_{4}= & d_{q p}-d_{p q}+\lambda_{A} \sum_{j \neq p, q} a_{j q} a_{j p}^{\prime}+a_{q j} a_{p j}^{\prime}-a_{j p} a_{j q}^{\prime}-a_{p j} a_{q j}^{\prime}
\end{aligned}
$$

To maximize (9) we form the Lagrangian under the constraint $c^{2}+s^{2}=1$ :

$$
L(c, s, \lambda)=k_{0} c^{2}+k_{1} s^{2}+k_{2} c s+k_{3} c+k_{4} s-\lambda\left(c^{2}+s^{2}-1\right) .
$$

First order optimality conditions yield:

$$
\begin{aligned}
2 k_{0} c+k_{2} s+k_{3} & =2 \lambda c \\
2 k_{1} s+k_{2} c+k_{4} & =2 \lambda s \\
c^{2}+s^{2} & =1,
\end{aligned}
$$

Solving this set of equations for $c$ we obtain:

$$
P_{c}(c)=\alpha_{4} c^{4}+\alpha_{3} c^{3}+\alpha_{2} c^{2}+\alpha_{1} c+\alpha_{0}=0,
$$

where

$$
\begin{aligned}
& \alpha_{4}=4 k_{2}^{2}+4\left(k_{0}-k_{1}\right)^{2}, \alpha_{3}=4 k_{3}\left(k_{0}-k_{1}\right)+4 k_{2} k_{4}, \\
& \alpha_{0}=k_{2}^{2}-k_{3}^{2}, \quad \alpha_{1}=4 k_{3}\left(k_{1}-k_{0}\right)-2 k_{2} k_{4}, \\
& \alpha_{2}=k_{4}^{2}-4 k_{2}^{2}+k_{3}^{2}-4\left(k_{0}-k_{1}\right)^{2} .
\end{aligned}
$$

Alternatively, we may solve for $s$, obtaining

$$
P_{s}(s)=\beta_{4} s^{4}+\beta_{3} s^{3}+\beta_{2} s^{2}+\beta_{1} s+\beta_{0}=0,
$$

where

$$
\begin{aligned}
& \beta_{4}=4 k_{2}^{2}+4\left(k_{1}-k_{0}\right)^{2}, \beta_{3}=4 k_{4}\left(k_{1}-k_{0}\right)+4 k_{2} k_{3}, \\
& \beta_{0}=k_{2}^{2}-k_{4}^{2}, \quad \beta_{1}=4 k_{4}\left(k_{0}-k_{1}\right)-2 k_{2} k_{3}, \\
& \beta_{2}=k_{3}^{2}-4 k_{2}^{2}+k_{4}^{2}-4\left(k_{1}-k_{0}\right)^{2} .
\end{aligned}
$$

The relation between the roots of these two polynomials is as follows:

$$
s=\frac{2 k_{2} c^{2}+k_{4} c-k_{2}}{\left(2 k_{0}-2 k_{1}\right) c+k_{3}}, \quad c=\frac{2 k_{2} s^{2}+k_{3} s-k_{2}}{\left(2 k_{1}-2 k_{0}\right) s+k_{4}} .
$$

To find the optimal $(c, s)$ pairs that maximize (9), we must root (13) or (15) and then make a substitution using (17) to obtain the $(c, s)$ pair. Unfortunately, (17) was derived supposing that the $(c, s)$ pair is a critical point of (9). If the computed roots are inexact, the evaluation of (17) will result in $c^{2}+s^{2} \neq 1$. Therefore, care must be taken to ensure that inaccuracies in the rooting of these polynomials does not result in non-orthogonal updates. We adopt the following method to circumvent this issue. Suppose that we have obtained a root $c$. To compute the corresponding $s$ while ensuring the orthogonality of the matrix $Q_{p q}(\theta)$, we find:

$s=\operatorname{sign}\left(s^{\prime}\right) \sqrt{1-c^{2}}$, where $s^{\prime}=\frac{2 k_{2} c^{2}+k_{4} c-k_{2}}{\left(2 k_{0}-2 k_{1}\right) c+k_{3}}$.

The next step is to evaluate the cost function for each obtained $(c, s)$ pair. The $(c, s)$ pair that results in the largest value of (9) is then used to perform the Jacobi update. Since the matrices $Q_{p q}(c, s)$ are sparse, these updates can be executed efficiently using standard Jacobi-update algorithms such as those found in [12].

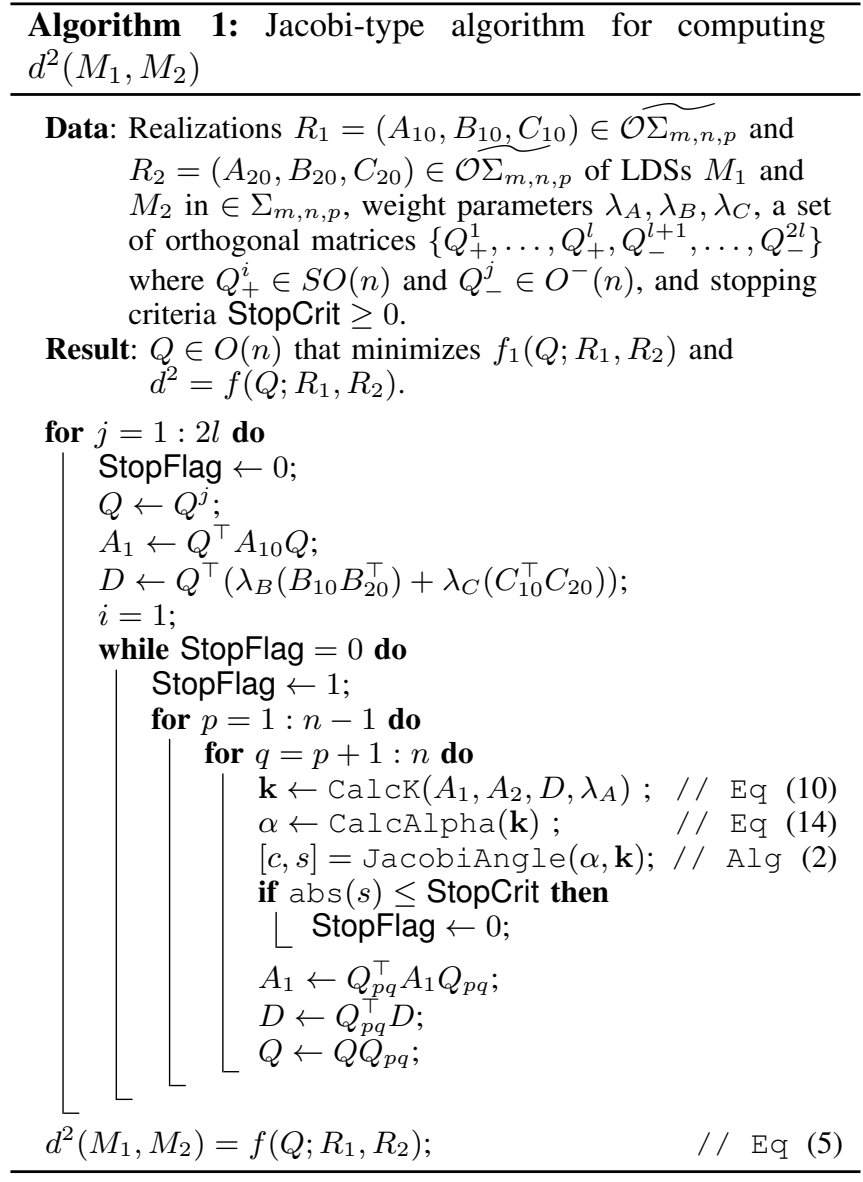




\section{B. Accurate and Fast Quartic Root Finding}

Obtaining the solutions to Equation (13) or 15$]$ in a fast and accurate manner is crucial to ensure the overall speed and accuracy of our algorithm. Interestingly, optimizing functions of the form (8) have appeared in the literature [8], most notably in the orthogonal joint diagonalization problem. To the best of our knowledge, the related fast and accurate rooting has not been addressed in the literature.

Our approach combines a closed form solution method with a Newton-Raphson root polishing step to obtain the roots of Equation (13) or 15). The first closed form solution method for quartics was derived by Ferrari in 1540 and is described in [1]. One benefit of such a formula is that it yields all four roots of the polynomial and hence we can, in principle, find global minimizers for our 1-dimensional minimization problems. Another benefit is in terms of speed: a straightforward C implementation of Ferrari's method is about 17 times faster than MATLAB's quasi-standard polynomial rooting function, which performs the QR algorithm on a polynomial's companion matrix. Nevertheless Ferrari's method is not popular [16] since it is highly prone to round off error. Strobach recently proposed a fast and accurate (general purpose) quartic root finding method based on iterative refinement of Ferrari's solution. Strobach's method is a viable option in our problem. However, in our application we can achieve the same accuracy as Strobach's method in better time. Note that since we are only interested in roots in $[-1,1]$ our problem is easier than general rooting (as Strobach's method). We simply use the roots returned by Ferrari's algorithm to initialize a Newton-Raphson root-polishing iteration. If the estimates of the roots are sufficiently accurate, a few iterations of Newton-Raphson improve their accuracy considerably as the number of significant digits nearly doubles at each iteration [11].

Another question is whether to solve for $\cos (\theta)$ or for $\sin (\theta)$. Strobach observed [16] that Ferrari's algorithm performs poorly in cases of large root spread, where this quantity is defined as the ratio of the maximal magnitude of a root to that of the minimal magnitude: $S=\frac{\left|r_{\max }\right|}{\left|r_{\min }\right|}$. As the algorithm converges $\theta_{p q} \rightarrow 0$ and hence at least one of the roots of the $P_{s}(s)$ polynomial approaches zero. In this case, the root spread becomes arbitrarily large. By solving the $P_{c}(c)$ polynomial, we avoid this situation. We have observed that Ferrari's method in combination with Newton-Raphson is about 3 times faster than Strobach's procedure, is easier to implement as it involves no parallel programming, and has comparable accuracy. Our method of computing Jacobi angles for our optimization sub-problem is summarized in Algorithm 2. In SIV, we present numerical evidence which confirms our claim that a Ferrari-based optimization which uses $\cos (\theta)$ is preferable (e.g., see Figure 1).

\section{Handling Local Minima}

Unfortunately, $f_{1}, f_{2}, f_{3}$ are not convex on $O(n)$ and they may have local minimizers which are not global. In fact, it is known that the only continuous convex function on a compact manifold is the constant function. Although we

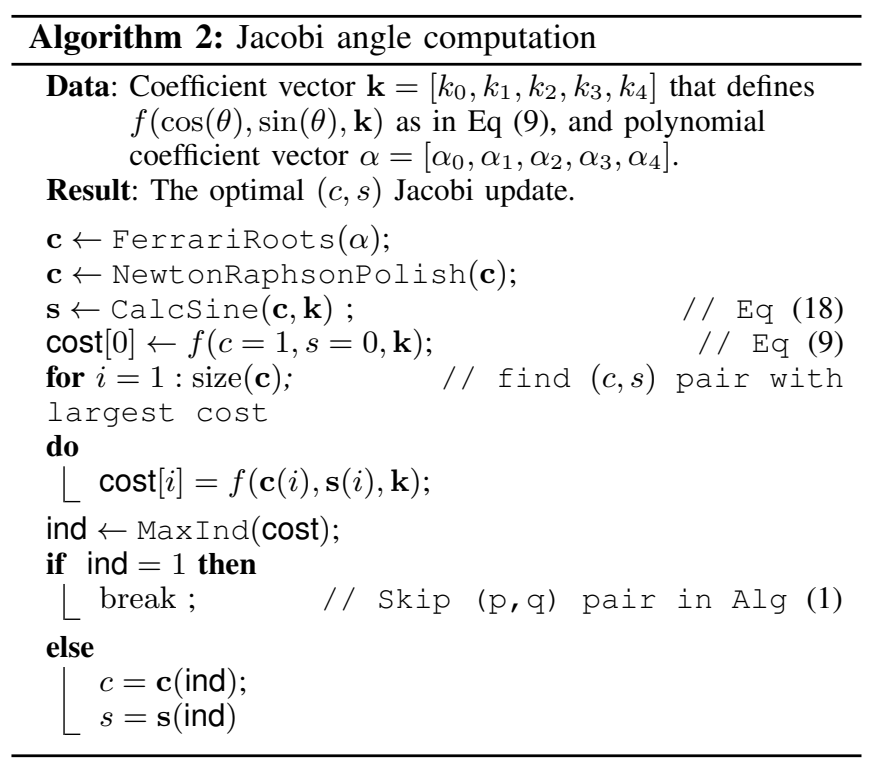

find global minimizers for the 1-dimensional sub-problems there is no guarantee that the algorithm will converge to the global minimizer of the main problem. A simple method to mitigate this difficulty is to begin the optimization with different initial matrices $Q$. For example, Algorithm 1 may be repeated with several random (uniformly distributed) initial points. Alternatively, the algorithm maybe repeated for a set of initial matrices $\mathcal{Q}_{+}^{l}=\left\{Q^{1}, \ldots, Q^{l}\right\} \subset S O(n)$ and $\mathcal{Q}_{-}^{l}=\left\{Q^{l+1}, \ldots, Q^{2 l}\right\} \subset O(n)$, where the matrices in each set are "maximally separated" in some sense. In fact, if such a set is available by simple function evaluations one may guess a good initial point and avoid running the algorithm $2 l$ times. One simple strategy to find the set $\mathcal{Q}_{+}^{l}$ is by maximizing an energy function such as

$$
E\left(\left\{Q_{i}\right\}\right)=\log \prod_{i \neq j}\left\|Q_{i}-Q_{j}\right\|_{F}^{2}=\sum_{i \neq j} \log \left\|Q_{i}-Q_{j}\right\|_{F}^{2} .
$$

Notice that a set $\mathcal{Q}_{-}^{l}$ can be found simply by swapping the first and second columns of each matrix in $\mathcal{Q}_{+}^{l}$. This energy function is known as the the logarithmic energy [10]. We can use a gradient method to find these matrices off-line (although this is not a completely satisfactory solution, since again we have no guarantee to find the global maximizers.) For $l=2$ a solution is simply $\mathcal{Q}_{+}^{2}=\left\{I_{n},-I_{n}\right\}$ if $n$ is even and if $n$ is odd $\mathcal{Q}_{+}^{2}=\left\{I_{n}, I_{n}^{-}\right\}$, where $I_{n}^{-}=$ $\operatorname{diag}(-1, \ldots,-1,+1)$.

\section{Comparison with Riemannian gradient descent}

A Riemannian gradient descent algorithm for minimizing $f\left(Q ; R_{1}, R_{2}\right)$ is presented in [4]. Each step of standard Riemannian gradient descent on $S O(n)$ involves computing the matrix exponential. Instead, one could use the polar decomposition (e.g., via the SVD) or the QR factorization (such maps are called retractions [2]) to reduce the computational cost of computing the matrix exponential. Both these approaches are of order $\mathcal{O}\left(n^{3}\right)$ (our experiments show that, as expected, the SVD-based method is several times slower 
but much more accurate). Moreover, to ensure decrease of the cost at each iteration one needs to implement a step-size rule such as Armijo's rule [2] which involves function evaluations and adds to the computational cost. On the other hand, a single sweep of our Jacobi-type algorithm requires $\mathcal{O}\left(n^{2}\right)$ updates. For each index pair, the computation of the coefficients in (10) and the Jacobi pair $(c, s)$ are of order $\mathcal{O}(n)$. Hence, the overall complexity of each step of our proposed Jacobi algorithm is $\mathcal{O}\left(n^{3}\right)$, which is the same complexity as Riemannian gradient descent. However, Jacobi-type methods have quadratic local convergence whereas the convergence for gradient descent is linear.

\section{NUMERICAL EXPERIMENTS}

In this section, we provide numerical experiments which show the efficiency and accuracy of our algorithm. To that end, we generate systems randomly. More precisely, we generate random realizations on a realizations space and this will induce random systems. We perform our experiments on $\mathcal{L}_{m, n, p}^{\mathrm{tC}, \mathrm{a}}$, the asymptotically stable subset of $\mathcal{L}_{m, n, p}^{\mathrm{tC}}$ (although the stability condition is not necessary as far as our computations are concerned). We choose $m=n=5$ and $p=10$. The space $\mathcal{L}_{m, n, p}^{\mathrm{tC}, \mathrm{a}}$ with large $p$ and $n \approx 5$ is the space which naturally appears in analysis of video sequence data (see [4]).

Data generation and algorithm settings. We want to generate two random systems $M_{1}, M_{2} \in \mathcal{L}_{5,5,10}^{\mathrm{tC}, \mathrm{a}}$, where $M_{2}$ is a perturbation of $M_{1}$. To this end, we generate random realizations $R_{1}, R_{2} \in \widetilde{\mathcal{O}}_{5,5,10}^{\mathrm{tC}, \mathrm{a}},\left(R_{i}=\left(A_{i}, B_{i}, C_{i}\right)\right)$ as follows: We first generate random matrices $S_{1}, \ldots, S_{5} \in$ $\mathbb{R}^{5 \times 5}$ and $T_{1}, T_{2} \in \mathbb{R}^{10 \times 5}$ with unit Gaussian elements. To generate a random stable matrix $A_{1} \in \mathbb{R}^{5 \times 5}$, we compute the matrix exponential $A_{1}=\exp \left(S_{1}-S_{1}^{\top}-S_{2} S_{2}^{\top}\right)$. This ensures that the magnitude of the eigenvalues of $A_{1}$ are less than 1 . We set $B_{1}=S_{3}$. To generate a random orthogonal matrix $C_{1}$, we compute the singular value decomposition (SVD) $T_{1}=U \Sigma V^{\top}$ and set $C_{1}=U$. To generate realization $R_{2}$ from $R_{1}$, we first generate a random orthogonal matrix $Q_{i} \in O(n)$. We then set $A_{2}=Q_{i}^{\top}\left(A_{1}+\sigma S_{4}\right) Q_{i}$ and $B_{2}=Q_{i}^{\top}\left(B_{1}+\sigma S_{5}\right)$. Likewise, to generate $C_{2}$, we set $C_{2}^{\prime}=\left(C+\sigma T_{2}\right) Q_{i}$. Since this matrix is not orthogonal, we compute the SVD, $C_{2}^{\prime}=U \Sigma V^{\top}$ and set $C_{2}=U$. Notice that if $\sigma=0$, then $R_{2}=Q_{i} \circ R_{1}$ (see (2)) and hence $M_{1}=M_{2}$. Now we generate $T=1000$ of such $\left(M_{1}, M_{2}\right)$ pairs with $\sigma \in\{0,0.2\}$, and run our Jacobi algorithm to find the distance $d_{1}\left(M_{1}, M_{2}\right)$ (with $\lambda_{A}, \lambda_{B}, \lambda_{C}=1$ ). We use initial sets $\mathcal{Q}_{+}^{2}$ and $\mathcal{Q}_{-}^{2}$ as in $\$$ III-C. We implement our Jacobi algorithm using MATLAB functions as well as an optimized MATLAB executable file (MEX file). Since our implementation of gradient descent also uses a combination of MATLAB and MEX functions, we consider our comparison to be fair.

Accuracy Comparison. The results for $\sigma=0$ are shown in Figure 11, which shows the point-wise median of the cost function (across $T=1000$ samples) in terms of the number of iterations (or sweeps) of the algorithms. We use the median simply to show a typical behavior. The results for both the Jacobi-type algorithm and the gradient descent (with SVD and Armjio step-size III-D are shown. Moreover, several possibilities for root-finding are examined, these include: Strobach+cos, Strobach+sin, Matlab+cos, Matlab+cos, Ferrari+cos, and Ferrari+sin. The Ferrari methods use at most 5 iterations of Newton-Raphson root polishing per root. Strobach + cos was not shown in this figure, as its performance was indistinguishable from Ferrari cosine. Since our implementation of Ferrari's method in Algorithm 2 is roughly three times faster than Strobach's general purpose quartic solver, we conclude that Ferrari+cos is the best among these root solving method for our Jacobi type algorithm. Figure 1 also shows that the difference in accuracy between Ferrari+cos and Ferrari+sin is significant. This difference in accuracy is much more pronounced for the Ferrari solver than for the Matlab and Strobach solvers, where the difference between using $\cos (\theta)$ and $\sin (\theta)$ is negligible (this justifies our choice of solving (13) instead of (15)).

Note that the Jacobi and gradient descent algorithms are both limited in the accuracy they may attain, since the minimum angles of rotation are limited by the machine epsilon. It appears that this limitation affects our Jacobi algorithm before affecting gradient descent. As both algorithms converge, the Jacobi algorithm will need to take smaller steps than gradient descent, as it is constrained to move in fixed directions and cannot move directly towards the minimum. As a consequence, it is expected that machine epsilon affects the Jacobi algorithm before affecting gradient descent. This explains the difference in maximal accuracy between the two methods.

Speed comparison. Figure 1 clearly shows superiority of our Jacobi-type algorithm compared with gradient descent in terms of number of iterations. Another speed comparison for the more realistic $\sigma=0.2$ scenario is given in Table II and in Figure 2, which shows the cumulative percentage of runs converged in terms of number of iterations (sweeps). We consider the algorithm converged whenever the algorithm reaches within $10^{-4}$ of the lowest value of $d_{1}^{2}\left(M_{1}, M_{2}\right)$ computed over 300 sweeps/iterations. Time per 1000 iterations / sweeps was measured on a HP Pavilion G7 laptop. The second column in Table I measures the average number of iterations needed to converge to $10^{-4}$ of the lowest cost for a given computation of $d^{2}\left(M_{1}, M_{2}\right)$. The third column is obtained by multiplying the first two. These results demonstrate that, in this specific example, our proposed Jacobi method converges roughly 20 times faster than Riemannian gradient descent.

Local minima. Next, we examine how algorithms perform when initialized only with one initial condition, namely, $\mathcal{Q}_{+}^{1}=\left\{I_{5}\right\}$ and $\mathcal{Q}_{-}^{1}=\left\{-I_{5}\right\}$. When $\sigma=0$ we consider an algorithm "converged to a global solution," if the final computed value is less than $10^{-4}$. In Table II, we see that using $\mathcal{Q}_{+}^{2}$ and $\mathcal{Q}_{-}^{2}$ improves global convergence when $\sigma=0$ for the gradient descent. However, interestingly, our Jacobi 


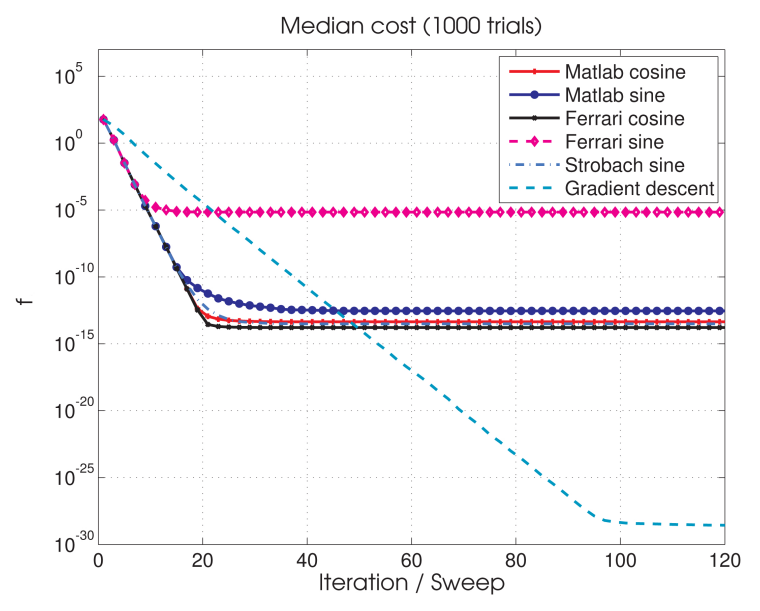

Fig. 1 - Typical convergence behavior for different algorithms and root finding methods $(\sigma=0)$.

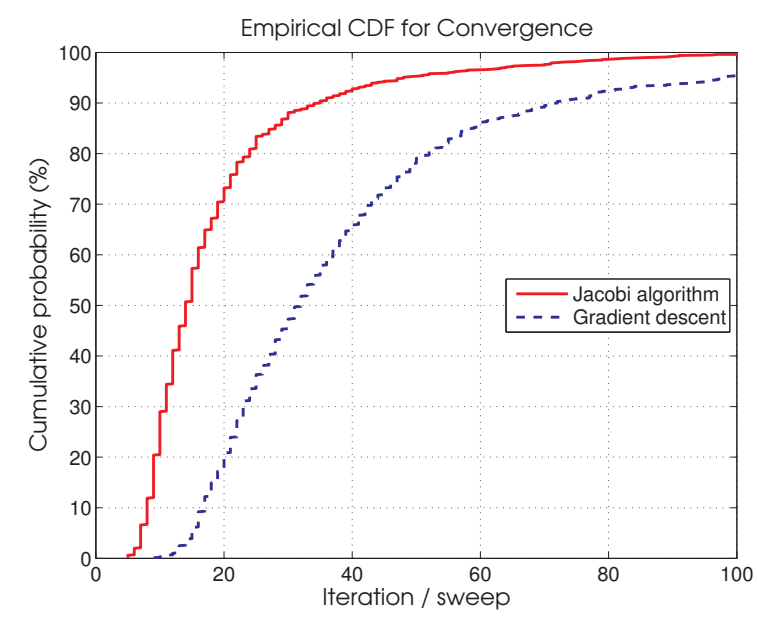

Fig. 2 - Empirical cumulative probability of convergence in terms of number of iterations $(\sigma=0.2)$.

algorithm already avoids local minima with $l=1$ and seems to be less propense to local minima than the gradient descent. This might be attributed to the fact that our Jacobi algorithm actually solves the 1-dimensional sub-problems globally.

\section{Discussion}

We have proposed a fast and accurate Jacobi-type algorithm for computing alignment distances between LDSs. Our algorithm is significantly faster than gradient descent for linear systems with orders that commonly arise in computer vision scenarios, and has comparable accuracy. Moreover, we hope that our insights will be useful in the investigation of other optimization problems involving orthogonality constraints.

Acknowledgements. This work has been supported in part by the grants ONR N00014-09-10084, NSF CAREER 0447739, NSF 0941362, NSF 0941463 and NSF 0931805.

\begin{tabular}{|l|c|c|}
\hline & Jacobi & Gradient descent \\
\hline Time per 10,000 sweeps / iterations & $0.50 \mathrm{~s}$ & $4.54 \mathrm{~s}$ \\
\hline Mean iterations to converge & 19.2 & 43.2 \\
\hline Mean time to converge & $1 \mathrm{x}$ & $20.43 \mathrm{x}$ \\
\hline
\end{tabular}

TABLE I - Speed comparison between gradient descent and our proposed Jacobi method for the $\sigma=0.2$ case.

\begin{tabular}{|l|l|l|}
\hline Number of initializations $l$ & 1 & 2 \\
\hline Jacobi global convergence (\%) & 100.0 & 100.0 \\
Gradient global convergence (\%) & 96.5 & 98.0 \\
\hline
\end{tabular}

TABLE II - Convergence of gradient descent to global minimizer(s) is enhanced by using two initializations $(\sigma=0)$.

\section{REFERENCES}

[1] M. Abramowitz and I. Stegun. Handbook of mathematical functions with formulas, graphs, and mathematical tables, volume 55. Dover publications, 1964.

[2] P.-A. Absil, R. Mahony, and R. Sepulchre. Optimization Algorithms on Matrix Manifolds. Princeton University Press, Princeton, NJ, 2008.

[3] B. Afsari. Simple LU and QR based non-orthogonal matrix joint diagonalization. In J. P. Rosca, D. Erdogmus, J. C. P., and S. Haykin, editors, ICA, volume 3889 of Lecture Notes in Computer Science, pages 1-7. Springer, 2006.

[4] B. Afsari, R. Chaudhry, A. Ravichandran, and R. Vidal. Group action induced distances for averaging and clustering linear dynamical systems with applications to the analysis of dynamic scenes. In IEEE Conference on Computer Vision and Pattern Recognition (CVPR), Providence, RI, 2012.

[5] B. Afsari and R. Vidal. Group action induced distances on spaces of high-dimensional linear stochastic processes. submitted.

[6] B. Afsari and R. Vidal. Group action induced distances on spaces of linear dynamical systems. submitted, 2013.

[7] B. Béjar, L. Zappella, and R. Vidal. Surgical gesture classification from video data. In N. Ayache, H. Delingette, P. Golland, and $\mathrm{K}$. Mori, editors, Medical Image Computing and Computer-Assisted Intervention - MICCAI 2012, volume 7510 of Lecture Notes in Computer Science, pages 34-41. Springer, 2012.

[8] J.-F. Cardoso and A. Souloumiac. Jacobi angles for simultaneous diagonalization. SIAM J. Mat. Anal. Appl., 17(1):161-164, January 1996.

[9] G. Doretto, A. Chiuso, Y. Wu, and S. Soatto. Dynamic textures. International Journal of Computer Vision, 51(2):91-109, 2003.

[10] P. Dragnev, D. Legg, and D. Townsend. Discrete logarithmic energy on the sphere. Pacific J. Math, 207(2):345-358, 2002.

[11] B. Flannery, W. Press, S. Teukolsky, and W. Vetterling. Numerical recipes in C. Press Syndicate of the University of Cambridge, New York, 1992.

[12] G. Golub and c. F. Van Loan. Matrix computations, volume 3. Johns Hopkins Univ Pr, 1996.

[13] U. Helmke and K. Hüper. A Jacobi-type method for computing balanced realizations. Systems \& Control letters, 39(19-30), 2000.

[14] P. S. Krishnaprasad. Geometry of Minimal Systems and the Identification Problem. PhD thesis, Harvard University, 1977.

[15] A. Souloumiac. Nonorthogonal joint diagonalization by combining givens and hyperbolic rotations. IEEE Transactions on Signal Processing, 57:2222-2231, June 2009.

[16] P. Strobach. The fast quartic solver. Journal of computational and applied mathematics, 234(10):3007-3024, 2010. 